Historic, Archive Document

Do not assume content reflects current scientific knowledge, policies, or practices. 



\title{
Wholesale Price - List
}

Hardy Vines, Shrubs, Etc.

\author{
SPRING AND FALL, 1917
}

\section{The PALISADES NURSERIES INCORPORATED}

\section{R. W. CLUCAS, Manager SPARKILL, N. Y.}

\section{INTRODUCTORY REMARKS AND TERMS}

\section{$T$}

HE plants offered herein are all sturdy, transplanted and nursery-grown for more than one year, and can be depended upon for flowering satisfactorily the first season after planting out.

Our stock of plants is reasonably large; we have thirty acres of hardy plants in cultivation. We make a specialty of supplying Landscape Gardeners, Dealers and others in the trade.

We print real prices, which, as long as unsold and without engagement, we are willing to accept. The prices are net, except being subject to 2 per cent discount when cash accompanies order; otherwise payable in thirty days from date of invoice on approved credit. Packing charges at cost, f.o.b. Sparkill, N. Y. To meet reputable competition, we will reduce our prices still further on being given the opportunity.

No less than six of one kind furnished at dozen rates, 25 at 100 rates, 250 at $\mathrm{I}, 000$ rates, unless otherwise specified. Clumps (or plants of extra-large size) can, in most cases, be supplied. Prices on application.

For varieties not listed herein see our Descriptive Catalogue of Palisades Popular Perennials. From prices quoted therein we allow the trade a special, liberal discount. 


\section{GENERAL LIST OF HARDY PERENNIALS}

\section{ACANTHUS}

Mollis. 4- and 5-in. Each Latifolius. 4 - and 5 - in pots................. 20

3-in. pots......... I0

\section{ACHILLEA}

Eupatorium, Parker's. IO Millefolium roseum... I0 Millefolium, Cerise Queen. New......... Ptarmica fl.-pl., The Pearl..............

Neige. New.........

I 00

\section{ACONITUM}

\begin{tabular}{|c|c|c|c|c|}
\hline Autumnal & 12 & I 25 & 9 & \\
\hline Fischeri.... & I 2 & I 25 & 9 & 00 \\
\hline Lycoctonum. & I5 & I 50 & Io & 00 \\
\hline Pyrenaicum. . & I5 & I 50 & IO & oo \\
\hline Napellus..... . & I 2 & I 25 & 9 & oo \\
\hline Napellus album...... & I 2 & I 25 & 9 & oo \\
\hline Napellus bicolor....... & I 2 & I 25 & 9 & 00 \\
\hline Napellus roseum ...... & I 2 & I 25 & 9 & 00 \\
\hline Sparks' Variety....... & I 2 & I 25 & 9 & oo \\
\hline Uncinatum.......... & Io & I 00 & 7 & 50 \\
\hline
\end{tabular}

\section{ACTEA}

See also, Cimicifuge

$\begin{array}{lllllll}\text { Japonica.............. } & 20 & 2 & 00 & 15 & 00 \\ \text { Spicata alba.......... } & \text { 10 } & 1 & 00 & 7 & 50\end{array}$ Spicata alba.

$\begin{array}{rrrrr}\text { Io } & \text { I } & 00 & 7 & 50 \\ \text { I0 } & 1 & 00 & 7 & 50\end{array}$

ADONIS

Vernalis........... I2 I $25 \quad 900$

Vernalis Amurensis... $40 \quad 400$

Vernalis Amurensis $40 \quad 400$

EGOPODIUM

Podograria variegatum Io I oo 750 AGROSTEMMA

$\begin{array}{llllll}\text { Coronaria............. } & \text { Io } & & 85 & 6 & 50 \\ \text { Coronaria alba....... } & \text { ro } & \text { I } & \text { o0 } & 7 & 50\end{array}$

AJUGA

$\begin{array}{lllllll}\text { Genevensis............ } & \text { Io } & \text { I } & \text { oo } & 7 & 50 \\ \text { Reptans atropurpurea. } & \text { Io } & \text { I } & \text { oo } & 7 & 50\end{array}$

Moly

\section{ALLIUM}

ALYSSUM

Sexatile compactum...

Sexatile compactum
fl.-pl., Gold Dust. 3-

in. pot-plants.

Rostratum

$\begin{array}{lllll}\text { I5 } & \text { I } & 50 & \text { 10 } & 00 \\ \text { I0 } & \text { I } & 00 & 7 & 50\end{array}$

AMAR YLLIS

Hallii (Lycoris squamig-

AMSONIA

Salicifolia............. Io I oo 750

ANCHUSA

Italica, Dropmore Variety. 3-in. potplants........... Io I oo 750 Strong field roots...... I 5 I 50 ro 00
Italica, Perry's Variety. Larger flowers and stronger grower. 3 -in.

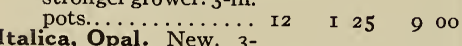
in. pot-plants....... I0 I 00 I 50 Strong field roots.......

Myosotidiflora. 3-in. 15 I 50 10 pots........... $20 \quad 2$ oo I5 00

ANEMONE JAPONICA

Alba, Honorine Jo-

bert. Single; white... Io I Io 750

Alice. Large; silvery rose I5 I 50 Io 50

Coupe d'Argent. New. I 2 I 25 I0 00

Geant des Blanches.

New...

I5 I 50 Io 00
ANEMONE JAPONICA, continued

Prince Heinrich. Large, Each Doz. 100 double; dark pink...\$0 Io $\$$ I oo $\$ 750$ Queen Charlotte.
Semi-double; day-

break-pink..... I $00 \quad 750$

Rosea superba. Single;

light rose.......... Io I $00 \quad 750$

Rubra............ Io I 00 Io 750

Whirlwind. Semi-dou-

ble; pure white...... Io I oo 750

Planting Stock of A. Japonica. Established plants of named sorts, ready May $\mathbf{I}$, in 2-in. pots, at half the prices quoted.

\section{ANEMONE, IN VARIETY}

Nemorosa (Wood Anem- Each Doz. 100 one) $\ldots \ldots \ldots \ldots \ldots$ io $\$$ I 00 \$7 50 Nemorosa fi.-pl. Double;

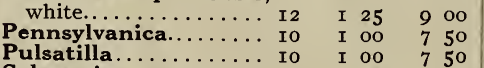

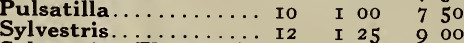

Sylvestris, Elise Fell-

man. Tall-growing;

large, double, white

flowers; continuous

bloomer.......... $20{ }^{2}$ oo I 500
Hepatica. See Hepatica triloba.

\section{ANTHEMIS}

Tinctoria Kelwayi..... Io I I $00 \quad 750$ Tinctoria Kelwayi alba Io 1 oo 750 Tinctoria Kelwayi

grandiflora. New.

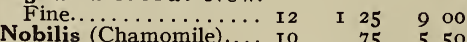

\section{ANTHERICUM}

$\begin{array}{llllll}\text { Liliago.................. Io } & \text { I } 00 & 7 & 50 \\ \text { Liliastrum major...... } & \text { I0 } & \text { I } & \text { oo } & 7 & 50\end{array}$

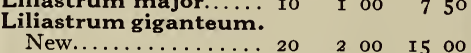

\section{AQUILEGIA}

Cærulea (True Rocky

Mountain Columbine)

Sky-blue, with white

$\begin{array}{llll}\text { corolla............ I } 2 & \text { I } 25 & 900 \\ \text { Carulea candidissima. } & & & \end{array}$

Carulea Hybrids. New.

Various colors; very fine, long spurs.

Cærulea Helenæ.

Alpina.............. I

Alpina superba. New. 25

Canadensis............ Io I

Chrysantha......

Chrysantha.........

Chrysantha grandiflora

alba. New.

Flabellata nana. Dwarf;

early; white.......... I

Nivea grandiflora. Large;
white............. I

Glandulosa major. Very fine variety.

Rose Queen. New. Rose

shades. Long spurs... I2

Skinneri............. I

Vulgaris. Blue . White.. I

Vulgaris alba. White..
Vulgaris fl.-pl. Double,

white; fine.......... Mixed................ Io

Single Varieties, Mixed.

Alpina compacta...... I

3 -in. pot-plants, or field I 2
Rosea............ 20

ARALIA

\section{Cashmeriana....... 20}

\section{ARENARIA}

Crspitosa........... I
Montana...... I2

\section{ARMERIA}

Laucheana.

Maritima splendens... Io

Maritima alba........ Io

Formosa hybrida. New.
Very fine............ Io

\section{ARTEMISIA}

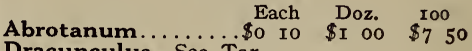
Dracunculus. See Tar-

ragon.

Stelleriana.......... Io I oo 750

Purshiana............... I0 10 Io 100

fine............... I5 I 25950

ASCLEPIAS

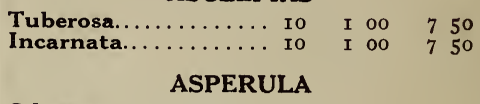

Odorata (Woodruff, or

Waldmeister)...... $20 \quad 200$

\section{ASPHODELUS}

Luteus............ I0 I $00 \quad 750$

\section{PERENNIAL ASTERS}

(Starwort, or Micholmas Daisios)

Price of strong plants that will make a profusion of bloom the first season planted, except where otherwise noted. Numbers 1 , 2,3 and 4, relative time of flowering.

Acris. (I) Bright lilac-purple flowers in great profusion; bushy habit. Height I ft. Acris nanus. Height 6 in.

Amethystinus. (3) Bright lilac flowers. Height $4 \mathrm{ft}$.

Beauty of Colwall. (I) New. Fine double blue. I2 cts. each, $\$ 1.25$ per doz., $\$ 9$ per 100.

Beauty of Ronsdorf. New. Large, lilacpink flowers; $21 / 2 \mathrm{ft}$. high. Continuous ploomer. $20 \mathrm{cts}$. each, \$I.75 per doz., \$I2 per roo.

Beaute Parfait. New. Large, dark violet flowers. Height $2 \mathrm{ft}$. I5 cts. each, \$I.5O per doz.

Chapniani (2) Light ageratum-blue; large sprays. Height $3 \mathrm{ft}$.

Collarette. (3) Blush-white. Height $3 \mathrm{ft}$.

Feltham Blue. (2) New. Large, pale blue flowers; free flowering and very showy. I5 cts. each, \$1.50 per doz., \$1o per Ioo.

Forget-me-not (Madame Seynouse). (2) This variety grows about 2 feet high, has strong, stiff stems, branched and covered strong, stiff stems, branched and covered
with myriads of small, daisy-like flowers; with myriads of small, daisy
white, with a brownish eye.

F. W. Raynor. (2) Light reddish violet.

F. W. Burbridge. (2) Large, soft heliotrope flowers; very fine. Height $4 \mathrm{ft}$.

Gertrude. (I) Foliage small and fine; habit graceful, smothered with a profusion of small, dainty, clear purple flowers.

Grandiflorus. (4) Best and largest; dark violet flowers; late and free bloomer. Height $21 / 2 \mathrm{ft}$. I $5 \mathrm{cts}$. each, $\$$ I.50 per doz., $\$$ Io per Ioo.

Janus. (2) Lavender flowers, with red cenJanus. (2) Lavender

Lævis. (3) Light heliotrope. Height $4 \mathrm{ft}$.

Lævis. (3) Light heliotrope. Height $4 \mathrm{ft}$. of rich lavender flowers. Height $4 \mathrm{ft}$.

Madonna. (2) Large; white.

Melpomene. (2) Tall; large, purple flowers in long, graceful sprays. Height $4 \mathrm{ft}$.

Mrs. Perry, Improved. (2) New. Large, rich rose-colored flowers. Height $3 \mathrm{ft}$.

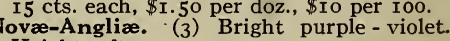
Height $4 \mathrm{ft}$.

Novæ-Angliæ rubra.

(2) Deep reddish

violet. Height 4

Novelty. (2) Small, deep pink flowers in great profusion.

Perry's Blue. (2) New. Large, rich lavender-blue flowers. Height $21 / 2 \mathrm{ft}$. I2 cts. each, \$I.25 per doz., \$9 per Ioo.

Perry's Favorite (Amellus). (2 and 3 ) New. Fine pink. I2 cts. each, \$I.25 per doz., $\$ 9$ per 100

Perry's Pink. (2) Rosy pink; mediumsized flower.

Peter's White. (2) New. Claimed by some to be the best white variety. Height $3 \mathrm{ft}$. I 5 cts. each, \$I.50 per doz., \$Io per Ioo. Ptarmicoides. (I) Small, pure white flowers; erect, dwarf habit. Height I ft. This is a continuous bloomer from July to frost. and makes a desirable border plant.

and makes a desirable border plant. Polyphyllus. (I) Elegant foliage; small,
white flowers with lemon centers. Very white flowers with lem
effective. Height $4 \mathrm{ft}$. 
PLEASE USE THIS ORDER BLANK

\section{The Palisades Nurseries}

\section{Sparkill, New York}

R. W. Clucas, Mgr.

Date

1917

Forward by $\left[\begin{array}{c}\text { Mall, Express } \\ \text { or Freight }\end{array}\right]$

Name

Street, P. O. Box

Rural Delivery

Post Office

County

State

\section{Express Office $\left[\begin{array}{l}\text { If different } \\ \text { from P. O. }\end{array}\right]$}

Fill orders will be forwarded as soon as possible after their receipt, unless we are tinctly, if you want the goods sent immediately; whether by mail or express, and when you want them thi

8. VERY IMPORTANT: Although you may have often written Office, County and State very plainly. By so doing you will avoid the possibility of delayand mistakes in filling your orders.

\begin{tabular}{|c|c|c|}
\hline $\begin{array}{l}\text { Quantity } \\
\end{array}$ & Prants, seeds or Balbs Wanteal & Dolatars \\
\hline & & \\
\hline & & \\
\hline & & \\
\hline & & \\
\hline & & \\
\hline & & \\
\hline & & \\
\hline & & \\
\hline & & \\
\hline & & \\
\hline & & \\
\hline & & \\
\hline & & \\
\hline & & \\
\hline & & \\
\hline & & \\
\hline & & \\
\hline & C & 1 \\
\hline
\end{tabular}

Amount Enclosed

$\$$

Please Leave Blank 


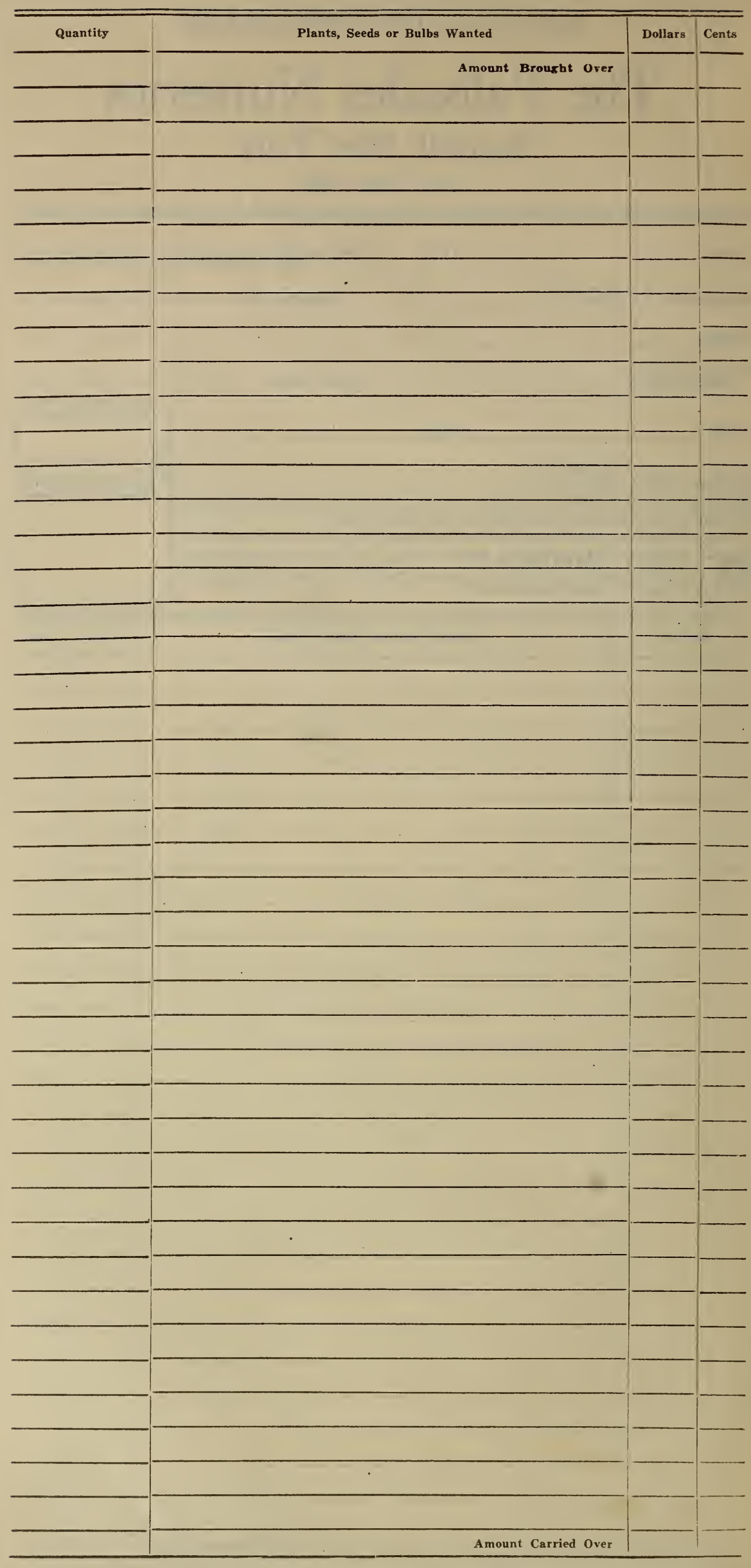




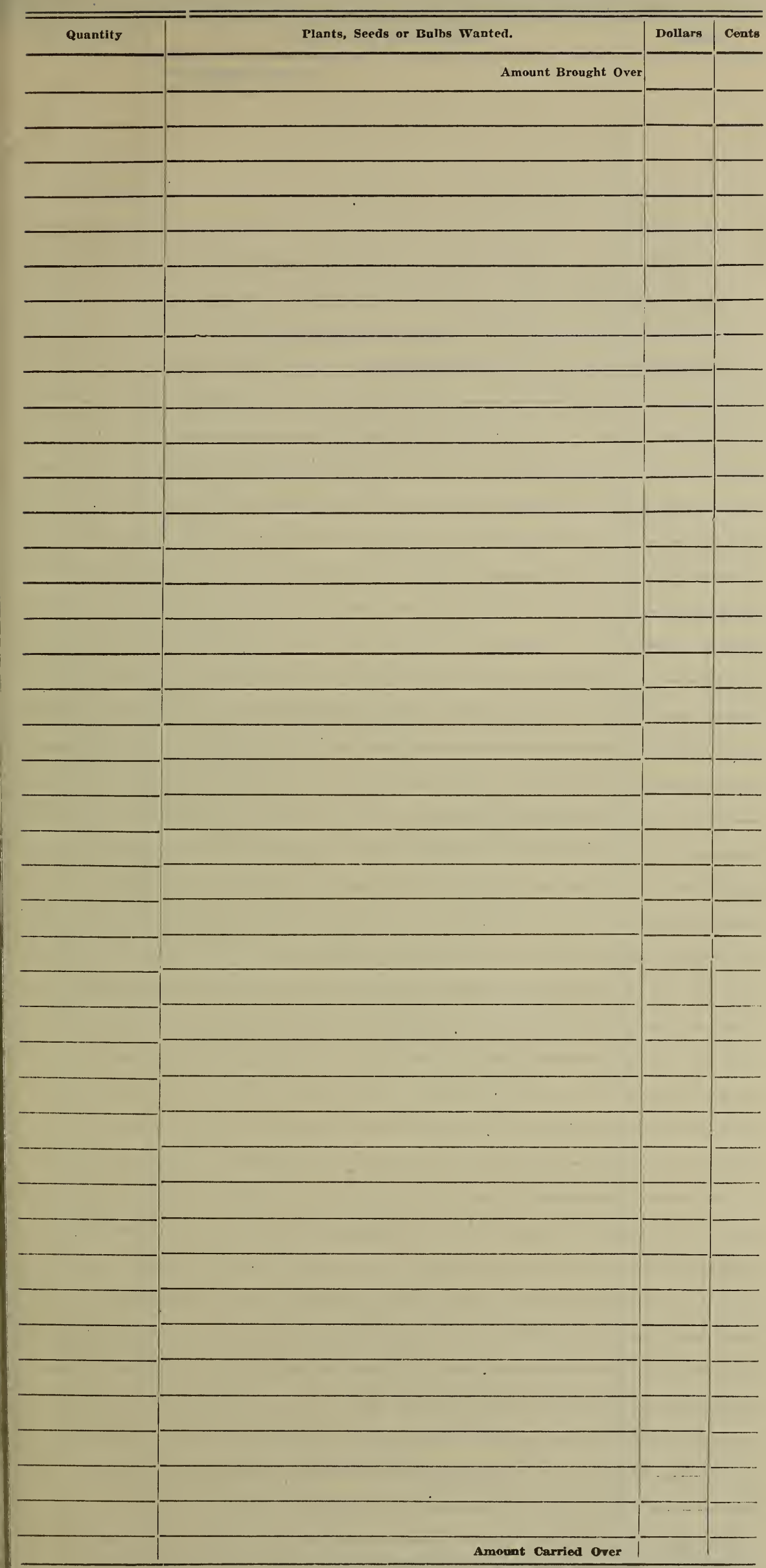




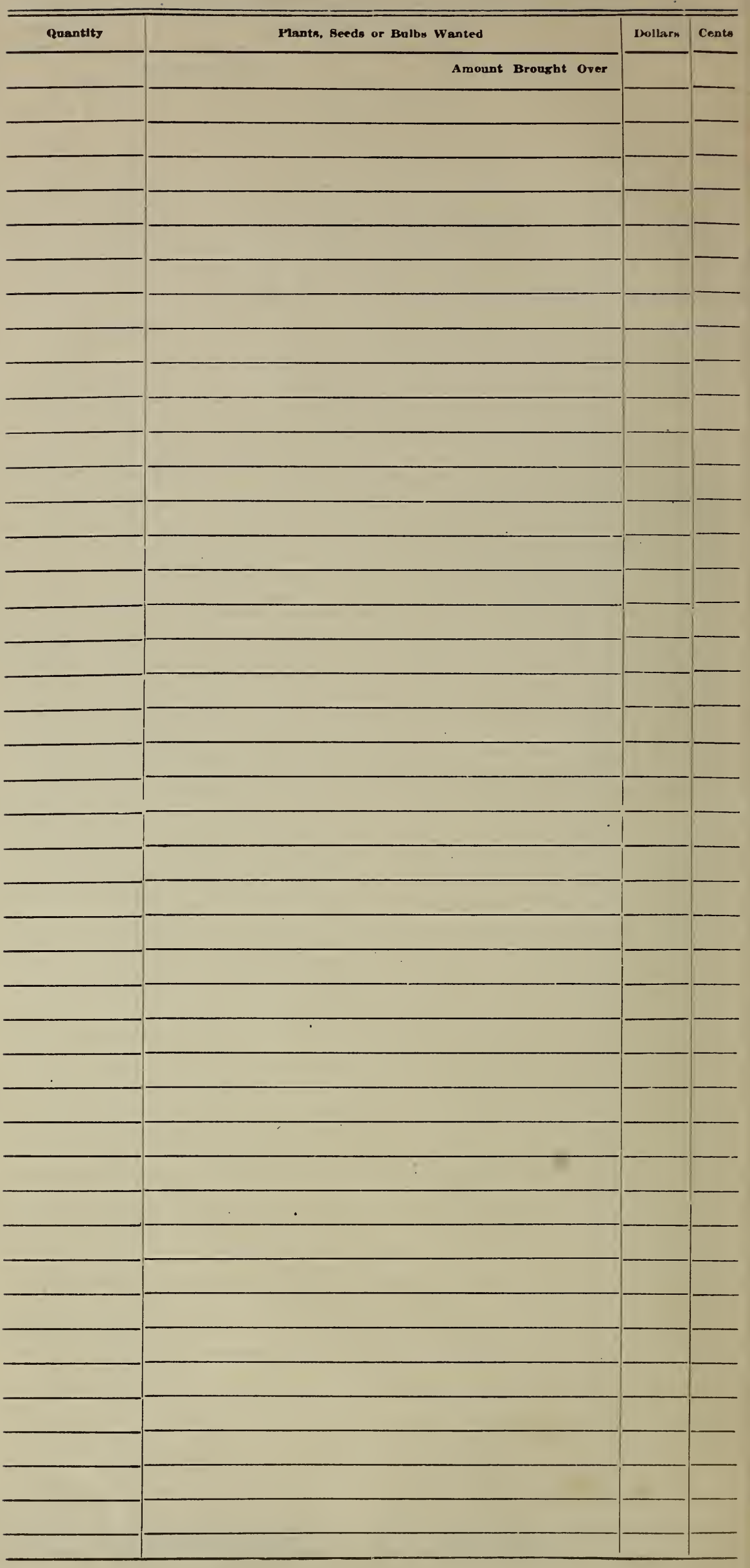


PERENNIAL ASTERS, continued

Robert Parker. (2) Large sprays of beautiful, large, soft lavender-blue flowers, with yellow centers; extra fine. Height $51 / 2 \mathrm{ft}$. A valuable cut-flower

Snowflake. (2) Pure white; very free. Height $I 1 / 2 \mathrm{ft}$.

Snow Queen. (2) Distinct, dwarf, white variety. Height $2 \mathrm{ft}$.

St. Egwin. (2) New. Clear pink; the best of all pink Asters. I5 cts. each, \$1.25 per doz., \$8.50 per roo.

Subcæruleus. (I) Very dwarf; blue daisylike flowers. May. I 2 cts. each, \$1.25 per doz., \$o per roo.

Tataricus. (4) Late; tall; blue. Height 5 to

Top Sawyer. (2) Large, lilac flowers of perfect shape. Height $4 \mathrm{ft}$.

Trinervius. (4) Rich violet-purple; distinct, large foliage. Height I to $2 \mathrm{ft}$.

large foliage. Height I to $2 \mathrm{ft}$.
T. S. Ware. (2) Light blue; one of the best of its color. Height $3 \frac{1}{2} \mathrm{ft}$.

Versicolor. (2) Small, white flowers, changing to rose; erect, branching habit. Height $31 / 2 \mathrm{ft}$

White Queen. (3) Large, white, free-flowering variety. Height 4 to $5 \mathrm{ft}$.

Wm. Bowman. (3) Large purple flowers on stems 3 feet high. A late, continuous bloomer; one of the latest to bloom, and has flowers equal in size to any.

ASTER-DWARF ALPINE VARIETIES

July-flowering; charming for rockwork Height 6 to 9 in.

Alpinus (Blue Moun-

tain Daisy). Flowers Each Doz. 100

bright purple.......\$0 Io $\$$ I $00 \quad \$ 750$

Alpinus albus. White... I0

Goliath. New hybrid.

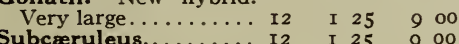

\section{ASTILBE}

Davidii. New and de-

sirable sort; graceful

flower; spikes of red-

dish purple. Height

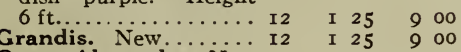

Queen Alexandra. New

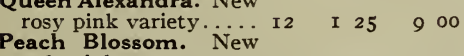

pale pink sort......... I2 I 25 I 00

For other varieties not listed under this

heading, see Spiræa, page 7 .

\section{ASTRANTIA}

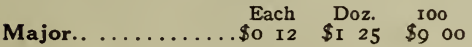
AUBRIETIA

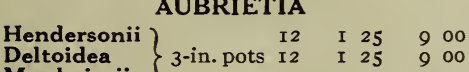

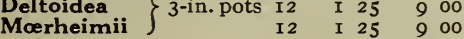

AURICULA

Alpina........... I2 I 25 9 00

BAPTISIA

Australis............ I0 I $00 \quad 750$

\section{BELLIS}

Perennis. Various colors ro I 00750

Grandiflora. New. Firstclass perennial....... I 2 I $25 \quad 800$ BOCCONIA

Cordata............ I0 I $00 \quad 750$ BOLTONIA

Asteroides............ I0 I oo 750

Latisquama...........

Latisquama nana. A
distinct, dwarf-growing

type; very fine........ I

BUDDLEIA

Veitchiana. New. Strong

field-grown plants..... 20 I 50 I2 00 3 -in. pot-plants.

3-in. pot-plants....... agnifica. New. 3-in. pot-plants.

Strong $\$ 50$ per $1,000 \ldots$ IO 4-in. pot-plants....... I5
BUPHTHALMUM

$\begin{array}{ccc}\text { Each } & \text { Doz. } & \text { I00 } \\ \text { Salicifolium......... } \$ 0 \text { IO } & \$ 1 \text { oo } & \$ 750\end{array}$

CACTUS, HARDY

See Opuntia.

\section{CALLIRHOE}

Involucrata......... I 2 I 259950

\section{CALTHA}

$\begin{array}{lllllll}\text { Palustris............... } & \text { Io } & \text { I } & 00 & 7 & 50 \\ \text { Palustris ff.-pl........ } & \text { I } 2 & \text { I } & 25 & 9 & 00\end{array}$

\section{CALYSTEGIA}

Pubescens plena. Per-

ennial; vine-like; a

double pink Morn-

ing - Glory or Gar -

denia.............. Io I 00750

\section{CAMPANULA}

Alliariæfolia.......... I 2 I 25

Carpatica, Blue........ I0 I 00

Carpatica, White....... Io I 00

Elegans............. Io I 00

Grossekii............. Io I 00 I0

Glomerata superba.

New................ I Latifolia macrantha...

Bells).
Medium, Blue.

Medium, Lilac.

Medium, White

Medium, Rose .........

Medium, Double-flow-

ered. Mixed colors...

Medium Calycanthema

(Cup-and-Saucer). Blue,

ors separate

Persicifolia, Blue.

I 2
I.
I 2
I 2

Persicifolia alba.

Persicifolia humosa.

Persicifolia grandifiora

Persicifolia Morheimi.

Consicifolia Morheimii.
Consider the finest

Considered the finest

Pyramidalis. Blue and

white.

Rapunculoides $\ldots \ldots \ldots$
Rotundifolia

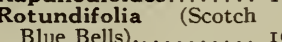

Blue Bells).......... I0 I $00 \quad 750$

Turbinata............ I5 I 50 I0

\section{CARYOPTERIS}

Mastacanthus. Field

plants............ I2 I 25 900

CASSIA

Marylandica......... I0 I $00 \quad 750$

\section{CATANANCHE}

Cærulea............ I0 I 00750

\section{CENT AUREA}

Montana alba........ Io I 00750 Montana rosea........ I 2 I $25 \quad 900$ Montana violetta...... Io I I $00 \quad 750$ Montana grandiflora.

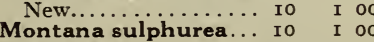

Dealbata............. I 2 I 25

Macrocephala.......... I0 I 100 750

Orientalis.............. I0 I0 I 00 I 7 50

CEPHALARIA

Tatarica (Alpina)..... I0 I $00 \quad 750$

CERASTIUM

Tomentosum........ I0 I 00 7 50

\section{CHELONE}

Glabra alba......... Io I

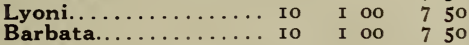

\section{CIMICIFUGA}

Racemosa (Serpentaria). Io I 00750
CHRYSANTHEMUMS

Various Type

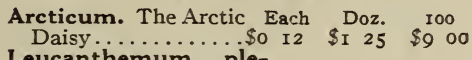

Leucanthemum ple-
num (Double Hardy

Marguerite). 21/2-in.

pots......................
Daisponicum (Shrubby.
See description

and illustration in de-

scriptive catalogue of

$\begin{array}{rlllll}\text { ennials.................... Io } & \text { I } 00 & 7 & 50 \\ \text { Inodorum(Bridal Robe). } & \text { Io } & \text { I } & 00 & 7 & 50\end{array}$

Uliginosum.......... I0 I

\section{HARDY}

\section{POMPON CHRYSANTHEMUMS}

Single and double; early and late-flowering

Our collection is a careful selection of what we consider the best of the standard sorts and newer introductions.

Price, strong pot-plants of varieties listed in our catalogue of Palisades Popular Perennials, on pages 10 and 11 , except where noted, 75 cts. per doz., $\$ 5$ per 100

Field roots, or clumps, for fall delivery, 10 cts. each, $\$ 1$ per doz., $\$ 7.50$ per 100. ]

\section{CHRYSANTHEMUM MAXIMUM}

(Giant Moonpenny or Shasta Daisies) ENGLISH or EUROPEAN VARIETIES

Mrs. J. Tersteeg. New, Each Doz. 100

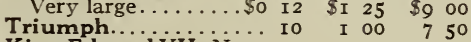

Kriumph.... Ving Edward New;

vigorous grower; enor-

mous white flowers... I2 I 25900 BURBANK VARIETIES

Alaska............. ro I California.......... Io I $00 \quad 750$ Stock (Burbank's).... Io $85 \quad 650$ Westralia............ I0 I 00 I0 750 CONVALLARIA

Majalis (Lily-of-the-Val$\begin{array}{llllll}\text { ley). Strong clumps... } & \text { I2 } & \text { I } 25 & & \\ \text { Crowns or pips....... } & \text { 05 } & 25 & \text { I } 50\end{array}$ COREOPSIS

Lanceolata grandiflora Io I $00 \quad 750$ Large clumps........ I 2 I 25 9 00

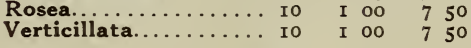
CRAMBE

Cordifolia.......... I2 I 25 9 00 CRUCIANELLA

Stylosa............ Io I $00 \quad 750$ CYPRIPEDIUM

Acaule............. $20 \quad 200$ I5 00 $\begin{array}{lllll}\text { Pubescens, per crown.. } 20 & 2 & 00 & \text { I } 500 \\ \text { Spectabile, per crown.. } 20 & 2 & 00 & \text { I5 } & 00\end{array}$

\section{DAPHNE}

Cneorum (Garland Flow-

er). First Size....... $25 \quad 2550 \quad 2000$

\section{DAISY}

Shasta. See Chrysanthemum maximum.

English. See Bellis perennis.

\section{DELPHINIUM \\ (Perential Larkour)}

Gold Medal Hybrids, Each Doz. 100

Finest Mixed.

Grown from seed

saved from the best

American and Euro-

pean strains and se-

lection made in our

Improved English $\mathrm{Hy}$ -

best named varieties.. $25 \quad 250 \quad 2000$

Belladonna........... I 2 I 25900

Chinense, Blue (Chi- Io I 00750

Chinense album

White........... Io I $00 \quad 750$

Formosum. Dark blue. Io I 00750

Light blue......... I 2 I 25900 


\section{DIANTHUS}

Barbatus (Sweet Wil- Each liam) - New port Pink ............\$o ro - Auricula-flowered... ro -Holborn Glory. Various colors.

-Double Crimson.......

-Double White........ 10

Deltoides alba...........

Latifolius atrococcin-

eus fi.-pl. (Hybrid William).

Plumarius (Hardy Pinks)

-Abbotsford. Deep rose Io

-Alice Lee. White.... I

Cyclops. Dark rose,

with crimson ring; very

with ; ingle flower..... Io

- Delicata. Delicate soft

rose; constant bloomer. I2

-Diamond. White.... Io

-Goliath. Very large

-Her Majesty. Double

white..

- Perpetuai Snow.

Everblooming white... I0

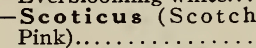

- Souvenir de Salle..... Io

-Mixed Colors..........

Superbus. Dwarf; white;

fine for cutting.......

-Fuerst Bismarck.

New.............. Io

scarlet............20

\section{DICTAMNUS}

Fraxinella. Red...... I 2

Fraxinella albus...... 12
DIELYTRA

Spectabilis......... I 2

Formosa, or eximia.... 12

DIGITALIS

Gloxiniæflora pur-

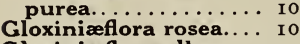

Gloxiniæflora rosea.... 10 I0

Gloxiniæeflora alba..... I0

Pale yellow........... I
Maculata superba (Iv-

aculata superba (Iv-
ery's Spotted). Spotted.

very fine............ ro

Monstrosa. Monster ter-

minal flowers.

DORONICUM

$\begin{array}{llllll}\text { Excelsum.............. I2 } & \text { I } & 25 & 9 & 00 \\ \text { Excelsum magnificum. } & \text { I2 } & \text { I } & 25 & 9 & 00\end{array}$ ECHINACEA

Helianthus......... I 2 I $25 \quad 900$

ECHINOPS

Ritro................ 10 I $00 \quad 750$ EPILOBIUM

Angustifolium....... I0 I $00 \quad 750$ EPIMEDIUM

Niveum........... I2 $\begin{array}{llll} & 25 & 9 & 00 \\ & & \end{array}$ ERIGERON

$\begin{array}{lllllll}\text { Glandulosus major.... } & \text { Io } & \text { I } & 00 & 7 & 50 \\ \text { Speciosus grandiflorus. } & \text { Io } & \text { I } & \text { oo } & 7 & 50\end{array}$ ERYNGIUM

Amethystinum. True

Blue Thistle.......... I2 I 25900

Planum.............. I I $_{\text {Holly) }}$ I 25 I 900

ERYSIMUM
Pulchellum.... I $25 \quad 900$

EUPATORIUM

Coelestinum............

Purpureum........... 10 $\begin{array}{rrrrr}75 & 5 & 50\end{array}$

EUPHORBIA

$\begin{array}{llllll}\text { Corollata................ } & \text { I0 } & \text { I } & 00 & 7 & 50 \\ \text { Polychroma.......... } & \text { I2 } & \text { I } & 25 & 9 & 00\end{array}$
CHOICE HARDY FERNS

Ferns are universally admired, and can be planted to beautify and make attractive odd, shady and unsightly corners. Below is a list adapted to this purpose.

Price, representing strong clumps of $\mathrm{va}$ rieties named below, 12 cts. each, $\$ 1.25$ per doz., \$9 per 100.

ADIANTUM pedatum (Maidenhair Fern). ASPIDIUM acrostichoides (Christmas Fern).

Marginale (Shield Fern).

Noveboracense (New York Fern).

Spinulosum (Evergreen Fern).

ASPLENIUM Filix-fœmina (Lady Fern).

CAMPTOSORUS rhizophyllus (Walking Fern).

CYSTOPTERIS bulbifera (Bulblet Bladder Fern).

DICKSONIA punctilobula (Gossamer Fern).

ONOCLEA.

Struthiopteris (Ostrich Fern).

Sensibilis (Sensitive Fern).

OSMUNDA cinnamomea (Cinnamon Fern).

Claytoniana (Interrupted Fern).

Regalis (Flowering Fern).

POL YPODIUM vulgare (Cliff Fern).

PTERIS aquilina (Eagle Fern). Evergreen.

\section{FEVERFEW}

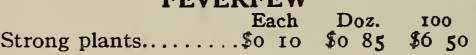

FRAGRARIA

Indica. Strawberry-lik ruit. Straw I 00750

\section{Riccartoni Hybrids (Hardy Fuchsia)}

FUCHSIA Strong plants.

\section{FUNKIA}

Albo-marginata....... I0 I $00 \quad 750$ Aurea variegata........ I0 I $00 \quad 750$ $\begin{array}{lllllll}\text { Minor alba............... } & \text { I0 } & \text { I } & 00 & 7 & 50 \\ \text { I } & \text { I } & 25 & 9 & 00\end{array}$

Sieboldiana. Fine blue foliage............ I2 I $25 \quad 900$

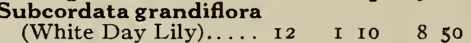
Undulata media variegata. The best variegated-leaf variety... Io I $00 \quad 750$

GAILLARDIA
maxima... Io

GALEGA

Officinalis. Blue or

White, each......... Io I oo 750

GALIUM
Mollugo........... I0 I 00 I0 750

GENTIANA
Andrewsii.......... I2 I 25950

GERANIUM
Grandiflorum........ I2 2 I 25 9 50

Sanguineum........ 12 I $\quad$ I 25 a 50

Atrosanguineum fi.-pl. ro I oo 750 $\begin{array}{lllllll}\text { Heldreichii............... } & \text { Io } & \text { I } & \text { oo } & 7 & 50 \\ \text { Heldreichii splendens. } & \text { I } & \text { I } & \text { oo } & 7 & 50\end{array}$ Magnificum........... ro I $100 \quad 7$ 50 Mrs. Bradshaw......... I 2 I 25 9 00

\section{GILLENIA}

Trifoliata.......... I 2 I 25900 GLAUCIUM

Luteum............ I0 I 00 , 50

GLECHOMA

See Nepeta

Trichosantha........ I0 I 00750 GYPSOPHILA

Acutifolia (Scorsoner
GYPSOPHILA, continued

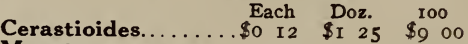
Monstrosa repens..... I 2 I $25 \quad 900$ Paniculata fl.-pl. New.

The double - flowering

form; very desirable... $20 \quad 200$ I5 00

GRASSES, Hardy Ornamental

All our Ornamental Grasses are nurserygrown, and large field clumps are sent on orders, unless otherwise specified.

ARUNDO Donax.

Strong dormant Each Doz. Ino

eyes............\$0 I5 \$I 50 \$10 00

Donax variegata..... I5 I 50 I 200

ARRHENATHERUM

bulbosum variega-

ornamental; striped

white and green; re-

tains its coloring late

in the season; quite

I 00750

BAMBUSA Metake.... $50 \quad 500$

ELYMUS glaucus..... I 12 I $25 \quad 900$

ERIANTHUS Ravennæ I0 I 00750

EULALIA Japonica.... Io Io I $00 \quad 750$

Japonica variegata... Io 10 I $00 \quad 750$

$\begin{array}{lllllllll}\text { Japonica zebrina.... Io } & \text { I } & 00 & 7 & 50 \\ \text { Gracillima univittata } & \text { I } & \text { I } & 00 & 7 & 50\end{array}$

FESTUCA glauca...... Io I 00750

GLYCERIA spectabilis foliis variegatis... I0 I 00750

GYNERIUM argenteum elegans (California

Pampas Grass).

Strong, 5-in. pots... $20 \quad 200$

PENNISETUM Japon-

icum............ I2 I 25900

PHALARIS arundi-

nacea variegata

Grass).............

tUMTHIUM robus-

\section{HELENIUM}

Autumnale super-

bum............. 10 I 00750

Autumnale rubrum... I 2 I 2590

Grandicephalum stri- Io I 00750

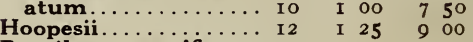

Pumilum magnificum.

$\begin{array}{lllll}\text { New................. I2 I } 25 & 9 & 00\end{array}$

\section{HELIOPSIS}

Pitcheriana.......... I0 I 00750 Pitcheriana semi-
plena

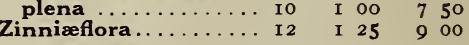
HELLEBORUS

Niger (Christmas Rose). $20 \quad 200$ I5 00 HELIANTHEMUM

Croceum.......... Io I oo 750

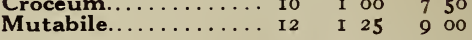

\section{HELIANTHUS}

H. S. Moon......... Io I oo 750 $\begin{array}{lllllll}\text { Maximiliani............ } & \text { IO } & \text { I } & 00 & 7 & 50 \\ \text { Mollis grandiflorus.... } & \text { Io } & \text { I } & 00 & 7 & 50\end{array}$

Multiflorus plenus.

Perhaps the best of the

hardy Sunflowers. Dou-

ble golden flowers in

great profusion, flower-

ing until late in the

season. 3-in. pots..... I0 I0 I $00 \quad 750$

Orgyalis.............. I0 I $\begin{array}{llllll} & \text { I } & 00 & 7 & 50 \\ 7 & 50\end{array}$

The Rev. Wolley Dodd. Io 1 oo $7 \begin{array}{llll}7 & 50\end{array}$

HELICHRYSUM

Angustifolium...... I 2 I 25900

HESPERIS

$\begin{array}{llllll}\text { Matronalis............. } & \text { Io } & \text { I } & \text { oo } & 7 & 50 \\ \text { Matronalis alba....... } & \text { Io } & \text { I } & \text { oo } & 7 & 50\end{array}$

HEPATICA

folia).............. Io I 00 , 50

Triloba........... Io I oo 750 
HEMEROCALLIS Each Doz. $\quad$ 100

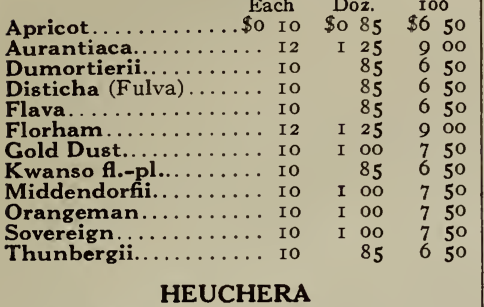

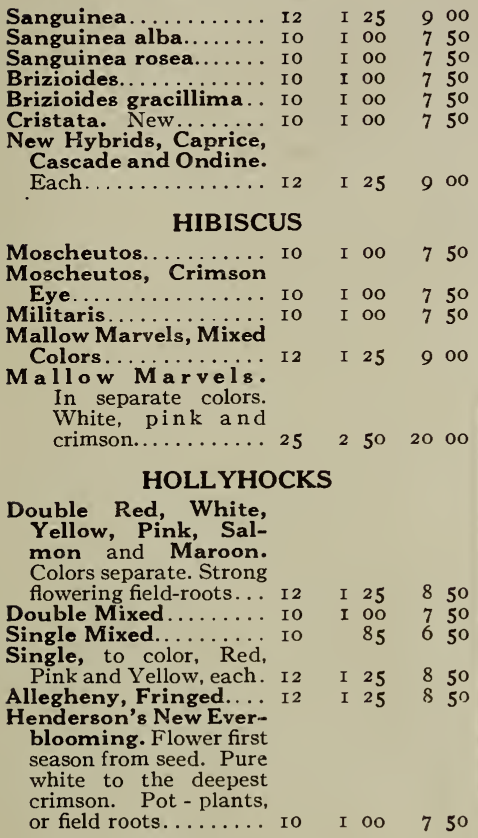

\section{HUMULUS}

pulus aureus (Gol-

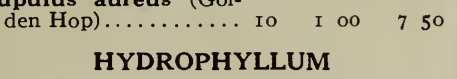

Canadense.......... I5 I 50 Io 00

\section{HYPERICUM}

Moserianum. 2-yr.-old

field-plants........... I 2 I $25 \quad 9$ oo $\begin{array}{rrrrrr}3 \text {-in. pots................ I0 } & \text { I } & 00 & 7 & 50 \\ \text { Moserianum tricolor. } & \text { I } 2 & \text { I } & 25 & 9 & 00\end{array}$ Adpressum............ I0 I

\section{IBERIS}

Sempervirens........ Io I 00750 Sempervirens, Snow 12 I 2590 $\begin{array}{cccccc}\text { Queen. New...... } & \text { I2 } & \text { I } & 25 & 9 & 00 \\ \text { Gibraltarica Hybrids.. } & \text { I2 } & \text { I } & 25 & 9 & 00\end{array}$ INCARVILLEA

$\begin{array}{llllll}\text { Delavayi.............. I2 } & \text { I } & 25 & 9 & 00 \\ \text { Grandiflora. New..... } & \text { I } 2 & \text { I } & 25 & 9 & 00\end{array}$

\section{INULA}

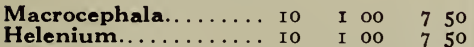

\section{IRIS GERMANICA}

(German Iris, or Fleur-de-Lis) For new varieties see Novelty Supplement
of Palisades Popular Perennials

Albino. Pale lavender; falls tipped with royal purple.

Charlotte Patty. Standards golden yellow; falls blue.

Chameleon. Delicate blue; falls tipped a little darker.

Fairy Queen. Upper petals white; lower veined with white.

Fantasy. Standards light blue; falls purple Florentina alba. Pure white. Early. Garrick. Standards light blue; falls a darker

Honorabilis. Golden yellow.
IRIS GERMANICA, continued

Kharput. Standards violet; falls violetpurple.

Madame de Brabant. Standards lavender; falls purple-streaked.

Madame de Baunne. Pure yellow.

Madame Chereau. White, feathered edges of sky-blue. Io cts. each, 75 cts. per doz., $\$ 5.50$ per I00, \$45 per I,000.

Mrs. Darwin. Standards pure white; falls veined purplish red. Io cts. each, \$I per doz., \$7.50 per Ioo.

Pauline. Light blue; lower petals slightly darker; flowers large.

Pallida (speciosa). Light blue.

Pallida Dalmatica. Extra-fine lavender colors. I5 cts. each, \$I.50 per doz., \$IO per I00.

Queen of May. Violet and heliotrope I5 cts. each, \$I per doz., \$7.50 per I00 Silver King. Pure white. Io cts. each, \$I Silver King. Pure white.
per doz., \$7.50 per I oo.

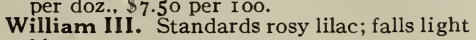
blue.

Price of strong, natural divisions of any of the above varieties, except where noted, 8 cts. each, 75

$\$ 45$ per 1,000 . doz., $\$ 3.50$ per $100, \$ 25$ per 1,000 .

IRIS, JAPANESE (Iris Kaempferi)

See our Descriptive Catalogue of Palisades Popular Perennials for names and descriptions; see also Novelty Supplement. Prices of clumps of 12 best double- and single-flowering named varieties, 15 cts. each, $\$ 1.25$ per doz., $\$ 9$ per 100 .

All colors mixed, $10 \mathrm{cts}$. each, $75 \mathrm{cts}$. per doz., $\$ 5.50$ per 100 .

\section{IRIS IN VARIETY}

Aurea (Golden Flag). Each Doz. 100 Distinct.......... \$o I2 $\$$ I 25 \$9 00

Enistata............. Io I

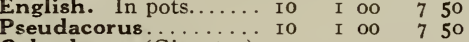

Ochroleuca (Gigantea). I5 I 50 Io 00

$\begin{array}{llll}\text { White and yellow..... I5 } & \text { I } 50 & \text { Io } 00 \\ \text { Orientalis, Snow Queen. } & & & \\ \text { Pure white. } & \text { I } 00 & 750\end{array}$

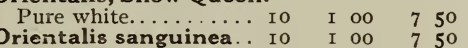

Orientalis sanguinea.

Maroon-red........... I0 I $00 \quad 750$

Pumila, Bride. Ivory-

white.............. Io I oo 750

Pumila cyanea. Deep

pumila, Fairy. Pale

blue.............. Io I oo 750 Intermediate hybrids. A new type, the re-
sult of crossing Germanica with Pumila manica
hybrid.

\section{Helge.}

Walhalla

Halfdan.

Ingeborg.
Sibirica alba

Tectorum

Tectorum.

JASMINUM

Nudiflorum. See Climbers.

\section{LAVANDULA}

Munsted. 2-inch pots.. Io I 00750 Vera (Sweet Lavender).. Io I 00750

\section{LATHYRUS}

Latifolius. Red....... I 2 I $\quad$ I $25 \quad 9900$

$\begin{array}{llllll}\text { Latifolius albus......... I2 } & \text { I } & 25 & 9 & 00 \\ \text { Latifolius, Pink Beauty. }\end{array}$ $\begin{array}{llll}\text { Very fine............ I } 2 \text { I } 25 & 900\end{array}$ New. White.......20 I 75

\section{LEONTOPODIUM}

Sibiricum (Siberian Edelweiss)........... I 2 I 25900 LIATRIS

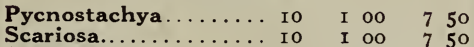

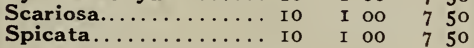

\section{LILIUM}

All herbaceous borders should have a few Lilies scattered through them, of which the following are the most satisfactory sorts to plant:
JAPANESE LILIES

Large-sized bulbs-

Auratum. Yellow and Each Doz. 100 white............. \$0 I2 \$I 25 \$9 00 Album (Speciosum).
White.................

elpomene (Speciosum) $20 \quad 2$ oO 12 oO

Rose...............

Deep red. ........ I2 I 25900

NATIVE AND OTHER HARDY LILIES

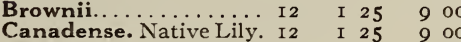

Canadense. Native Lily. I2 I 2590

tion Lily). 4-in. pots. I I I 2590

Croceum........... I2 I $25 \quad 900$

$\begin{array}{lllllll}\text { Superbum } \ldots \ldots \ldots \ldots \ldots & \text { IO } & \text { I } & 00 & 7 & 50 \\ \text { Tenuifolium } & \ldots \ldots \ldots & \text { I } 2 & \text { I } & 25 & 9 & 00\end{array}$

Tigrinum splendens...

$\begin{array}{llllll}\text { Tigrinum fi.-pl......... } & \text { I0 } & \text { I } & 00 & 7 & 50 \\ \text { Umbellatum......... } & \text { Io } & \text { I } & \text { oo } & 7 & 50\end{array}$

\section{LINUM}

Perenne. Blue........ I0 I $00 \quad 750$ $\begin{array}{llllll}\text { Perenne album. White. } & \text { IO } & \text { I } & \text { oo } & 7 & 50 \\ \text { Flavum.............. I } 2 & \text { I } & 25 & 9 & 00\end{array}$

LOBELIA

Cardinalis. 3-in. pots.. Io I $00 \quad 750$ LUPINUS

Polyphyllus. Blue.... I 2 I 25900

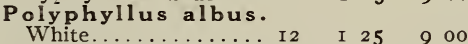
$\begin{array}{lllll}\text { Polyphyllus roseus.Rose I } 2 & \text { I } & 25 & 9 & 00 \\ & 25 & 9 & 00\end{array}$

\section{LYCHNIS}

Chalcedonica.......... I0 I oo 750 Chalcedonica carnea.. Io I 00750
Chalcedonica fl.-pl. New.............. I2 I 25900 Semperflorens plena... I0 I I $\begin{array}{lllll}\text { Semperflorens plena... I } 2 & \text { I } & 25 & 9 & 00 \\ \text { Viscaria splendensfl.-pl. IO } & \text { I } & \text { OO } & 7 & 50\end{array}$ LYCORIS

Squamigera. See Amaryllis.

LYSIMACHIA

Clethroides.
Nummularia

LYTHRUM

Roseum superbum.... I0 I $00 \quad 750$

Roseum superbum,
Perry's Variety. New;

fine sort.

I 2 I $25 \quad 900$

MALLOW MARVELS. See Hibiscus. MALVA

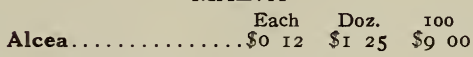

MEGASEA. See Saxifraga.

MENTHA

Spicata (Spearmint).... 10 $10 \quad 75 \quad 550$ $\begin{array}{lllll}\text { Riperita (Peppermint).. Io } & & 75 & 5 & 50 \\ \text { Pulegium (Pennyroyal). I } & \text { I } 25 & 9 & 00\end{array}$ Viridis. Variegated. Io $\begin{array}{rrr}75 & 550 \\ \text { Requieni. Creeping... I } 2 & \text { I } 25 & 9\end{array}$ MERTENSIA

Virginica.......... IO I $00 \quad 750$ MONARDA

Didyma alba ........ I 2 I $25 \quad 900$

Didyma rosea.......... Io I Io 750

idyma splendens,

Violacea superba...... I0 Io I oo $7 \begin{array}{llll}7 & 50\end{array}$

$\begin{array}{lllllll}\text { Russelliana. Pale blue. } & \text { Io } & \text { I } & \text { oo } & 7 & 50 \\ \text { Ro } & 7 & 50\end{array}$

Fistulosa alba......... I0 I 100

MYOSOTIS

Palustris semperflorens. Io I 00750 Palustris semperfiorens,

grandiflorus ........ I 2 I $25 \quad 900$

NEPETA

Glechoma hederacea

$\begin{array}{cccc}\text { folia variegata..... Io } & 85 & 650\end{array}$

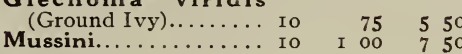




\section{ENOTHERA}

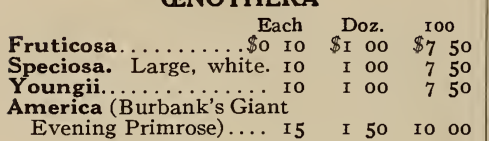

\section{OMPHALODES}

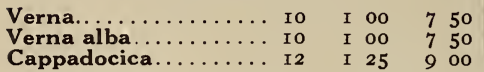

ONOPORDON

Robert Bruce. New... I0 I oo 750

$$
\text { OPUNTIA }
$$

Rafinesquii (Hardy Cac-

tus)............. Io I oo 750

\section{PACHYSANDRA}

$\begin{array}{llllll}\text { Terminalis. Strong, } 2- & & & & \\ \text { yr. field-plants. } & \ldots & 20 & \text { I } 75 & \text { I } 2 & 00\end{array}$ $\begin{array}{rrrrrrr}\text { yr. field-plants........ } & 20 & \text { I } & 75 & \text { I } 2 & 00 \\ 3 \text {-in. pot-plants....... } & \text { Io } & \text { I } & \text { oo } & 7 & 50\end{array}$

\section{PAEONIA SINENSIS}

\section{(Double Chinese Peony)}

NAMED VARIETIES

See our Descriptive Catalogue of Popular Perennials for names and descriptions. PRICES of named varieties of doubleflowering Chinese Peony roots:

Strong root-divisions with 2 or 3 eyes,

20 cts. each, $\$ 2$ per doz., $\$ 15$ per 100 .

Strong root-divisions with 3 to 5 eyes,

25 cts. each, $\$ 2.50$ per doz., $\$ 20$ per 100 .

Strong whole roots, 2 years from divided

stage, $40 \mathrm{cts}$. each, $\$ 4$ per doz.

Strong whole roots, 3 years from divided

stage, $65 \mathrm{cts}$. each, $\$ 6.50$ per doz.

\section{PAEONIA SINENSIS, MIXED COLORS}

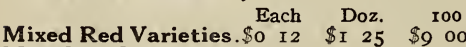
Mixed Pink Varieties.. I 2 I $25 \quad 900$ Mixed White Varieties. I 2 I 25 9 00 Mixed, All Colors...... I 2 I 2590

\section{SINGLE PEONIES}

Single and Semi-dou-

Single and Semi-dou- 30

Named, 10 Separate
Sorts............ $40 \quad 400$

\section{PAEONIA OFFICINALIS}

Officinalis $\mathbf{r} \mathbf{u} \mathbf{b} \mathbf{r} \mathbf{a}-\mathbf{p l}$.

Old double red. Large

handsome, rich crim-$$
\text { son. }
$$

Officinalis rosea-pl. Old

double rose. Full rose,

passing off flesh-color. I5 I 50 I0 00

Mutabilis alba-pl. Old

double flesh-white.

Flowers charming pink

passing off white.

I 5 I $50 \quad$ I2 00

\section{PAONIA TENUIFOLIA}

(The Fennel-leaved Peony)

Double-flowered........35 350

\section{JAPANESE TREE PEONY}

Extra-strong plants, Io distinct, double varieties, each \$I.

\section{PAPAVER ORIENTALE}

\section{Giant Oriental Poppy}

Named Sorts from Cuttings-Not Seed-

For names and descriptions see our Descriptive Catalogue of Palisades Popular Perennials.

Prices of named varieties:

3 -inch pots (in spring only), $10 \mathrm{cts}$. each,

$\$ 1$ per doz., $\$ 7.50$ per 100

4 -inch pots, 12 cts. each, $\$ 1.25$ per doz.,

$\$ 9$ per 100 .

The large size is certain to bloom the first year.

For fall delivery we supply field roots only at 15 cts. each, $\$ 1.25$ per doz., \$9 per 100 .

Mixed Colors, Seedlings of Oriental Poppies.

Strong plants from 3-

in. pots, in spring Each Doz. 100 Field plants, for spring or fall delivery...... Io I $\quad$ oo 750
PAPAVER NUDICAULE

\section{(Alpine Poppies)}

Mixed Seedlings. All Each Doz. 100

colors.............. \$o Io \$I oo $\$ 750$

Separate Colors. White

\section{PARDANTHUS}

Chinensis........... 10 $85 \quad 650$

\begin{tabular}{|c|c|c|}
\hline \multicolumn{3}{|c|}{ PENTSTEMON } \\
\hline Barbatus Torreyi..... Io & I 00 & \\
\hline Digitalis. & 85 & \\
\hline Grandiflorus & I 25 & 900 \\
\hline Ovatus... & I oo & 750 \\
\hline Pubescens. & I 00 & 750 \\
\hline Tubiflorus & I 25 & 900 \\
\hline
\end{tabular}

\section{PHLOX, PERENNIAL}

Those marked with an asterisk (*) are the varieties we consider the best for all purposes.

NOTICE.-Our prices are for large 2-yearold field-grown plants.

\section{PHLOX DECUSSATA, or PANICULATA}

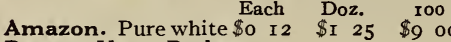

Baron Van Dedem.

New. Large flower,

almost blood-red..... I 2 I 259900

Bridesmaid. Pure white

with rich crimson eye. Io I oo 750

*aran d'Ache. Cerise-

pink; showy and free

flowering............

purplish crimson; fine

for massing..........

* Coquelicot. Scarlet.... I

Daybreak. New. Pale
pink...............
Dr. Charcot. Violet-

white center..........
claireur. Carmine -

Eclaireur. Carmine -
purple............. I

*Etna. Orange-scarlet...

Elizabeth Campbell.

Eugene Danzanvililiers.

Lilac and white......

Fernand Cortez. Deep

F. G. Von Lassburg.

Gine pure white.....

*General von Heutze.

New brilliant salmon-

red, white center;

Gruppenkonigin. Pale

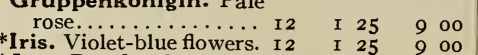

*Jean Barth. Soft clear pink; center cherryred.

La Vogue. Mauve.

Le Mahdi. Purple.... . I

L'Esperance. Laven-

der-pink, white center.

New. Paul Dutrie.

New. Delicate lilac-

* Matador. O............

*Mrs. E. E. Jenkins.

Pure white, enormous
truss; very fine flower

and free bloomer.....

rose..............

Currant-red, with a

Rijnstroom. New.

Strong stem; rosy pink. Io I oo 750 P. Struthers. Rosy

red eye............. Io I oo 750

* Selma. Delicate rose,

with deep rosy eye;

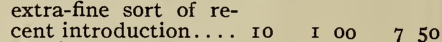

Sheriff. Ivory, salmon,

buff............. I2 I $25 \quad 900$

Tapis Blanc. New

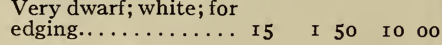

PHLOX DECUSSATA IN MIXTURE

In going over our Phlox during the growing season, we find many sorts sporting, which are always carefully removed and thrown together in mixture. Besides, names are sometimes lost by accident. This mixture
PHLOX DECUSSATA, continued contains some of the best types of all colors, making them suitable for massing where no particular color effect is desired. Strong, field-grown roots, in mixture, 60 cts. per doz., $\$ 4$ per I00, $\$ 35$ per 1,000 .

ARENDSI DECUSSATA CANADENSIS

A new race of Phlox, flowering in May on strong stems, I2 to 24 inches high; blooms persistently for more than two months.

Amanda. Delicate lilac.

Charlotte. Rosy lilac.

Grete. Ivory-white.

Helene. Pale blue.

Luise. Lilac, red eye.

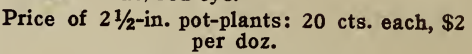

\section{PHLOX SUFFRUTICOSA}

(Early-flowering Phlox)

These flower two or three weeks before Decussata, and make fine spikes of bloom again in the fall. Specially useful for cutting.

Enchantress. New. Each Doz. 100

Shell - pink. Same

habit as Miss Lin-

gard............. \$o I2 \$I 25 \$9 oo

Forward. Violet flow-

ers striped white..... Io I
* Indian Chief. Clear

purple-red; rich shade. Io I $00 \quad 750$ Miss Lingard. Pearly

cate pink eye with deli-

cate pink eye. Very

remarkable bloomer,

producing two or three

crops of flowers during

the season; indispen-

sable as a cut-flower

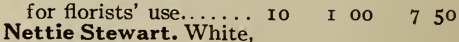

with light pink eye... Io I oo 750

Ringleader. White.

large violet-red eye.. Io I oo 750

PHLOX SUbUlata (Dwarf Moss Pinks)

Strong field clumps, or plants from 3 -inch pots.

Alba. White........\$o Io \$o 75 \$5 50

Alba. White........ \$o Io $\$ 075 \$ 550$
Bride. White, with crim-

son eye............. Io I oo 750

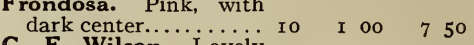

G. F. Wilson. Lovely

$\begin{array}{rrrrr}\text { mauve.............. Io } & \text { I } & \text { oo } & 7 & 50 \\ \text { Lilacina. Light lilac... } & \text { Io } & 75 & 5 & 50\end{array}$

Nelsonii. Dwarf; com-

$\begin{array}{rrrrr}\text { pact; white.......... } & \text { I } 2 & \text { I } 25 & 9 & 00 \\ \text { Rosea. Bright rose..... } & \text { I0 } & 75 & 5 & 50\end{array}$

OTHER VARIETIES (Types) OF PHLOX

Amœna............. Io I $00 \quad 7$ oo

Amcena folia variegata.

lavender Phlox. $2^{1} / 2$-in

pots ............ $20 \quad 200$ I5 00

Divaricata alba gran

diflora............20 2000

Divaricata Canadensis.

Perry's Variety. New.
Pewicata

Perry's Variety. New. I 5 I 50

Ovata Carolina (Laurel-

Leaf Phlox)......... Io I 00 is 50 PHYSALIS $\begin{array}{llllll}\text { Franchetii.............. } & \text { Io } & \text { I } & \text { oo } & 7 & 50 \\ \text { Bunyardii........... } & \text { Io } & \text { I } & \text { oo } & 7 & 50\end{array}$ PHYSOSTEGIA

Virginica. Pink...... I0 $85 \quad 650$ $\begin{array}{lllll}\text { Speciosa. Bright rose... } & \text { Io } & 85 & 6 & 50 \\ \text { Speciosa alba. White.. } & \text { Io } & 85 & 6 & 50\end{array}$ PLATYCODON $\begin{array}{llllll}\text { Grandiflorum album.. } & \text { Io } & 85 & 6 & 50 \\ \text { Grandiflorum, Blue... } & \text { Io } & 85 & 6 & 50\end{array}$ $\begin{array}{llllll}\text { Grandifiorum, Blue... } & \text { Io } & 85 & 6 & 50 \\ \text { Mariesi macrantha.... } & \text { Io } & 85 & 6 & 50\end{array}$ PLUMBAGO

Larpentx.......... I0 I 00750 PODOPHYLLUM

Peltatum.......... Io I oo 750 POLEMONIUM

Cæruleum........... Io Io I 2590 Cæruleum album..... I0 I0 I 25 9 00 POLYGONATUM

Majus............ ro I oo 750 


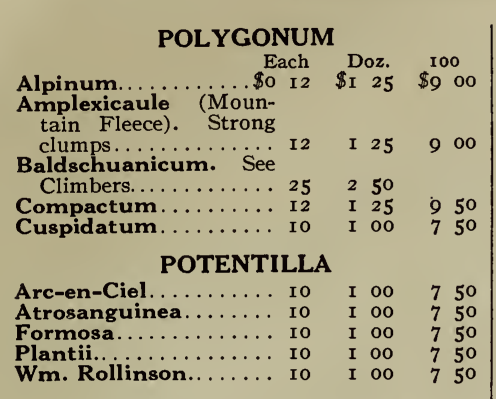

PRIMULA (Hardy Primrose)

Acaulis fimbriata. Va-

rious colors........... I2 I $25 \quad 9$ o0

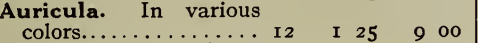

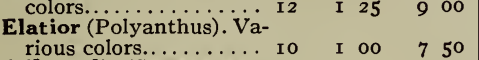
rious colors............ Io
Officinalis (Sweet-scent-

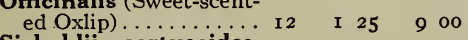
Sieboldii cortusoides. Four colors........... I2 I 25 I 00 Veris (English Cowslip). Io I oo 750 Veris superba. Giant Yellow Polyanthus. True............. I2 I $25 \quad 950$ Veris superba fi.-pl. (Hose-on-Hose, or Doulow Polyanthus). New.

Fine for florists...... I5 I 50 Io 00

Vulgaris (English Yellow

Primrose). True im-

ported stock......... Io I Io 750

PRUNELLA
Webbiana............ I0 I oo 750
PULMONARIA

$\begin{array}{llllll}\text { Saccharata maculata.. } & \text { I } 2 & \text { I } & 25 & 9 & 50 \\ \text { Angustifolia. Azurea.. } & \text { I2 } & \text { I } & 25 & 9 & 50\end{array}$ PYRETHRUM, Single Varieties

The brilliant and fresh colors of the Single Pyrethrum render it a desirable plant for cutflowers for market.

Roseum hybridum

grandiflorum. Su-

perb, new, large, sin-

gle-flowered varieties Each Doz, Ioo in mixture, all colors. \$o I0 $\quad \begin{array}{llll}\text { I } & \text { oo } & \$ 7 & 50\end{array}$

Hybridum fl.-pl. Dou-

ble-flowered; in four

best named varieties.

Red, White, Pink, and

Yellow. 3 -in. pots.... $25 \quad 2 \quad 50 \quad 20$ oo

Little Gem............ I0 I $\begin{aligned} & \text { I } \\ & \text { oo }\end{aligned}$

Uliginosum (Giant

Daisy)............... Io I oo $\quad 750$
Nipponicum. See Chry-

santhemum.......... Io I

\section{RANUNCULUS}

Aconitifolius ff.-pl..... I2 I 25 I 900 $\begin{array}{llllll}\text { Repens f.-pl............. } & \text { IO } & \text { I } & \text { oo } & 9 & 50 \\ \text { Rpeciosus fl.-pl........ I } 2 & \text { I } & 25 & 9 & 00\end{array}$ RHEUM
Palmatum.......... I5 I 25 I 00

Palmatum........ I5
RHEXIA
RH

Virginica............ I0 $\quad 75 \quad 550$ ROMNEYA

Coulteri (California Tree Poppy). We have a nice stock of this most showy and popular perennial, and can offer strong plants in the following sizes at these attractive prices:

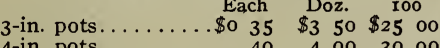
4 -in. pots. Extra-strong field roots 50

\section{ROSMARINUS}

Officinalis (Rosemary).

$$
\text { In 3-in. pots........... I } 00 \quad 750
$$

\section{RUDBECKIA}

Nitida, Autumn Glory.

Tall, single variety;

large, yellow flowers

from August to Octo-

Maxima. Very attrac-

....... I0 I $00 \quad 750$

Nowmanii............. 10 I 100 is
RUDBECKIA, continued

Purpurea ( $\mathrm{P}$ u r p l e Each Doz.

Cone-flower).......\$0 Io $\$$ I oo $\$ 750$ Subtomentosa......... I0 IO I oo 750 Golden Glow (Lacini-

$\begin{array}{lrrrrr}\text { ata). Clumps......... } & \text { Io } & \text { I } & \text { oo } & 7 & 50 \\ \text { Strong divisions....... } & \text { o5 } & 50 & 3 & 50\end{array}$

SAGINA

Glabra.............. Io I oo 750

SALVIA

Argentea............ I0 I0 I oo 750 $\begin{array}{lllllll}\text { Azurea grandiflora..... } & \text { IO } & \text { I } & 00 & 7 & 50 \\ \text { Greggii. } 3 \text {-in. pots..... } & \text { I } 2 & \text { I } & 25 & 9 & 00\end{array}$ SAGE

Holt's Mammoth. $21 / 2$ -

$\begin{array}{lllll}\text { in. pots............... } & \text { 05 } & 50 & 3 & 50 \\ \text { Large field clumps.... I0 } & 75 & 5 & 50\end{array}$

SANTOLINA

Incana. Field plants... I0 $\quad \begin{array}{r}75 \\ 5\end{array}$

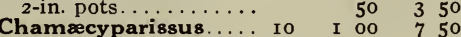

SAPONARIA $\begin{array}{llllll}\text { Ocymoides splendens.. } & \text { Io } & \text { I } & \text { oo } & 7 & 50 \\ \text { Caucasica fl.-pl......... } & \text { Io } & \text { I } & \text { oo } & 7 & 50\end{array}$ SAXIFRAGA

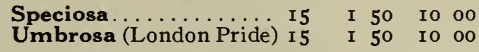
SCABIOSA

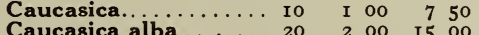
Caucasica alba .......... 20 20 2000

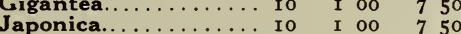

\section{SCUTELLARIA}

Baicalensis coelestina.. I2 I 2590 SEDUM

Acre. Very dwarf...... Io

Maximum album..... Io

Maximowiczii. Vellow. Io

Spectabile.

Spectabile, Brilliant... I
Spectabile atropurpu-

reum.

Spurium coccineum....

Sexangulare.

Reflexum.

Oppositifolium.......... I

\section{SEMPERVIVUM}

Arachnoideum........ I0 I 00

Arachnoideum........ 10 10 $100 \quad 750$

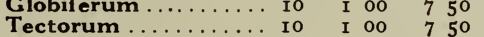

SENECIO

Clivorium. New. Intro-

$\begin{array}{rlllll}\text { duced from China....... } & \text { I2 } & \text { I } & 25 & 9 & 00 \\ \text { wilsonii............... I2 } & \text { I } & 25 & 9 & 00\end{array}$

\section{SHASTA DAISY}

See Chrysanthemum maximum.

SIDALCEA

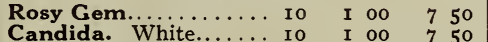
SILENE

Asterias............. Io I oo 750

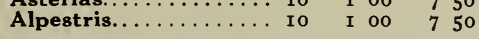

SMILACINA
Racemosa........... Io I oo 750 SOLIDAGO

Canadensis........... 10

(1)

SPERGULA

Pilifera............. I5 I 50 I0 00 SPIRAEA

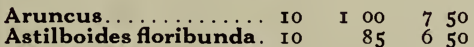
Chinensis............. 10 I0 1 1 $00 \quad 750$ Filipendula ff.-pl....... 10 Japonica............. Io Japonica compacta

multifiora.

Palmata.

Palmata elegans....... I0 I $100 \quad 750$

Ulmaria aurea f...pl... I0 I2 I 120 I 2550

Venusta........... I2 I 25

For other varieties, see Astilbe. STATICE

$\begin{array}{lll}75 & 5 & 50 \\ 85 & 6 & 50\end{array}$
STACHYS

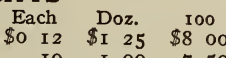

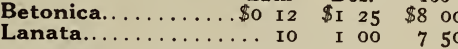

STOKESIA

Cyanea. Lavender-blue

flowers, 4 to 5 inches in

diameter............ Io I $00 \quad 65^{\circ}$

Cyanea alba. New white

form of the above..... I0 I 00650

SWEET WILLIAM

See Dianthus barbatus.

SYMPHYTUM

Aureum............ Io I oo 750

TANACETUM

Balsamita (Costmary, or $\begin{array}{rlllll}\text { Bible Leaf)............ } & \text { Io } & \text { I } & \text { oo } & 7 & 50 \\ \text { Vulgare (Tansy) ....... } & \text { Io } & \text { I } & \text { 0o } & 7 & 50\end{array}$

TARRAGON (ESTRAGON)

$\begin{array}{lllll}\text { Mailing size ........... I0 } & 75 & 5 & 50 \\ \text { Field Clumps......... I } & \text { I } & 25 & 8 & 50\end{array}$ TEUCRIUM

Chamædrys.......... I0 I oo 750

THALICTRUM

Aquilegifolium album. I2 I $25 \quad 900$

Adiantifolium......... I2 12 I 259900

Dioicum. Purple flowers I2 $\begin{array}{llll}25 & 9 & 00 \\ & & \end{array}$

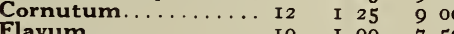

Glaum.............. 10 10 10 750

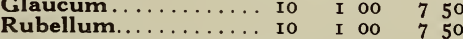

\section{THERMOPSIS}

$\begin{array}{llllll}\text { Caroliniana........... I2 } & \text { I } & 25 & 9 & 00 \\ \text { Fabacea............... I2 } & \text { I } 25 & 9 & 00\end{array}$ THYMUS

Serpyllum (Creeping io I oo 750

Lanuginosus (Woolly-

leaded Thyme)...... Io I $00 \quad 750$

(Lemon Thyme)..... Io I oo 750

Vulgaris (English Thyme) Io \begin{tabular}{lllll} 
I & oo & 7 & 50 \\
\hline
\end{tabular}

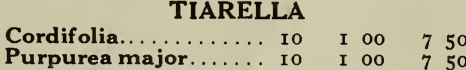

\section{TRADESCANTIA}

Virginiana. Blue...... 10 I $00 \quad 750$ TRICYRTIS

Hirta........... I2 I 259900 TRILLIUM

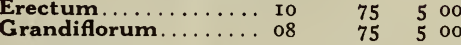
TRITOMA (Red-Hot-Poker Plant)

Express. New variety;

very early flowering;

light and dark orange

flowers...............
Pfitzerii. The everbloom-

ing Tritoma, with flow-

ers of rich orange-

$\begin{array}{llll}\text { scarlet............... Io I } & \text { I } \\ \text { Rufa. Showy spikes of } & & & \end{array}$

deep yellow flowers

with crimson anthers.

Fine new variety..... I2 12 I $25 \quad 9$ o0

Tuckii (May Queen).... I0 I $125 \quad 900$

Uvaria grandiflora.
Late flowering........ I0 $85 \quad 650$

Small-sized plants, in pots, suitable for mailing or planting out (all will flower the flrst year) of the above varieties of Tritomas, except Rufa, $\$ 3.50$ per $100, \$ 30$ per 1,000 .

TUNICA

Saxifraga........... $\begin{gathered}\text { Each } \\ \text { Doz. }\end{gathered}$ \$I oo $^{100}$ TROLLIUS

Europæus............ I5 $\quad$ I $25 \quad 900$ Sinensis Ledebourii... 20 20 200 15 00

VALERIANA

Coccinea.............. I0 I $00 \quad 750$

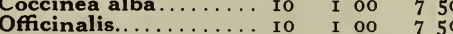
Montana...............

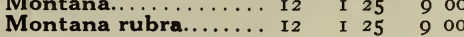




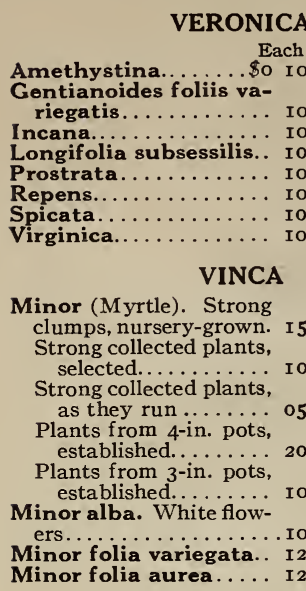

VIOLA (VIOLETS)

Odorata. Semper flo- Each Doz. 100 rens. Seedling plants.\$0 Io \$I $00 \quad \$ 750$ dorata, La France.

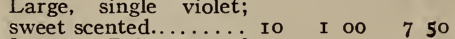

sweet scented.........
Odorata, Princess of

Wales. Broad, pansy-

like flowers of deep

violet color........... Io I $00 \quad 750$
Odorata, Hardy Double

English. Fine deep

purple; very fragrant.. Io I oo 750
Pedata (Bird's-foot

Violet)............ Io I $00 \quad 750$

VIOLA CORNUTA (Tufted Pansy)

Cornuta alba......... I5 I 25900

Cornuta, Admiration.

Dark blue............ Io I $00 \quad 650$
Cornuta lutea splen-

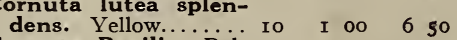

$\begin{array}{lllll}\text { Cornuta, Papilio...Pale } & \text { Io } & & 6 & 50 \\ \text { blue............ Io I } 00 & 6 & 50\end{array}$

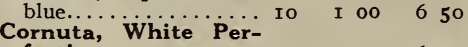

VIOLA CORNUTA, continued

Cornuta, G. Wermig, or Purpurea. New. Numerous dark blue flowers on long stems closely resembling violets. A really good thing for the florist. A summer violet of real interest. Each Doz. Ioo $21 / 2$-in. pots........ \$0 I0 \$0 85 \$6 50 Gracilis............. I I 25 9 00

\section{WALLFLOWER}

English, Double or Single. Strong, I-yr.-old

plants, suitable for early

forcing. 4 -in. pots..... I 2
Cloth of Gold. Bright

canary-yellow; single

YUCCA

Filamentosa. Fine, large,

2-yr.-old plants, from

open ground.... from I2 I 25900

Extra-strong, 3-yr.-old

plants.

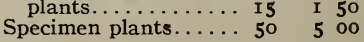

\section{HARDY VINES AND CLIMBERS}

\section{ACTINIDIA}

Polygama. Extra- Each Doz. 100 strong, 3 -yr.-old
plants ............ $\$ 0 \quad 25 \quad \$ 2 \quad 50 \$ 2000$ AKEBIA

Quinata. Strong,

old plants......... I2 I I 25 I 900 AMPELOPSIS

Veitchii. Extra-strong, 3 -ft. vines, 3-yr.-old $20 \quad 200$ is 00 roots, XXX,......... yr.-old plants, XX.. I5 I 50 10 00 Strong, 2-yr.-old, fieldgrown roots, with vines I ft.long, X. Io $\quad 75 \quad 550$

Muralis. Strong, 3-yr. old plants, heavy... I 2 I 25900

Quinquefolia (Virginia Creeper). 3-yr.-old plants, extra heavy.... Io I 00700 Engelmanii. Strong, yr.-old plants, heavy.. I 2 I 25900

\section{ARISTOLOCHIA}

Sipho (Dutchman's Pipe).

$\begin{array}{llllll}\text { Extra heavy........... } & 50 & 4 & 00 & 30 & 00 \\ \text { Strong, 2-yr.-old plants } & 20 & 2 & 00 & 15 & 00\end{array}$ APIOS

Tuberosa (Groundnut). Strong tubers...

\section{BIGNONIA}

Grandiflora. Extra-strong plants............. 25
Strong, 2-yr.-old plants

Strong, 2-yr.-old plants
Radicans. Extra-strong plants.

Strong, 2-yr.-old plants CELASTRUS

Scandens. 2-yr.-old

plants............20 200 I5 00 CINNAMON VINE

Strong plants......... 06 $40 \quad 250$

\section{CLEMATIS}

LARGE-FLOWERING VARIETIES

Duchess of Edinburgh. Double; white. Henryi. Finest white.

Jackmanii. Royal purple.

Mme. Baron Veillard. Light rose.

Mme. Edouard Andre. Rich, velvety red.

Ville de Lyon. New. Red flowers.

Strong 2-year-old plants, above named 72 rietios, 35c. oach, $\$ 3.50$ per doz.

SMALL-FLOWERING VARIETIES

Paniculata (Virgin's Each Doz.

Bower). No. I, XX

strong, 3-yr.-old

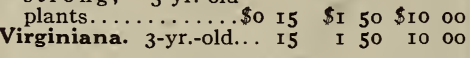

Radicans. Evergreen
climber. Strong, 3-yr.-

old field-grown plants. I5 I 50 Io 00

Radicans variegata. Va-

riegated leaves. 3 -in.

pot-plants.......... Io I $00 \quad 750$

trong, 3-yr.-old field

plants........... I5 I 50 Io co

Radicans vegetus.

Strong 2-yr. field plants I5 I 50 Io 00

\section{HUMULUS}

Lupul

(Perennial

Hop).......... $05 \quad 50 \quad 350$

Hop). Strong....... Io I 00750

HONEYSUCKLE (LONICERA)

Aurea reticulata. Yellow-variegated foli-

age.
Belgica. Yellow flowers

Evergreen (Sempervirens). Red flowers.

Halliana. Pure white, changing to buff.

Extra-strong, field-grown plants of above

four varieties, 15 cts. each, $\$ 1.50$ per doz. $\$ 12$ per 100 .

\section{IVY}

True Hardy English-not the So-called. Field-grown, strong grade, 2 - to 3 -ft. vines.
KUDZU VINE

See Pueraria. Plants Each Doz. 100 from 3-in..pots.... \$O IO \$1 00 \$7 50

\section{JASMINUM}

Nudiflorum (Hardy

Yellow Jasmine).

yr.-old plants......... I5 I 259900

Beesianum. New Red

\section{LATHYRUS}

Latifolius (Everlasting

Sweet Pea). Red..... I 2 I 25900

Latifolius albus. White. I 2 I 25900

Latifolius, Pink Beauty.

Rose. Fine for cutting. I 2 I 25900

Latifolius, White Pearl 20

LYCIUM

Barbatum (Matrimony

Vine). Strong....... I2 I 259900

Extra-strong, 4-yr.-old

\section{POLYGONUM}

$B$ aldschuanicum

multiflorum. New.

Hardy white climber;

rapid grower. Strong,

field-grown plants..... I5 I 50 I0 00

Plants from 3 -in. pots. Io $85 \quad 650$

Auberti. New. 2-yr.

field plants........ $25 \quad 250 \quad 2000$ 3 -in. pots..........

\section{PUERARIA}

Thunbergiana (Kudzu

Vine, or Jack-and-the-

Beanstalk). 3-in. pots. Io I $00 \quad 750$

Field grown........ I5 I 50

\section{WISTARIA}

Sinensis. Blue, or White.

Strong 2-yr.-old plants. $20 \quad 200$ I5 00 3 -yr.-old plants....... $35 \quad 3 \quad 50 \quad 2500$

\section{SURPLUS LARGE-SIZED SHRUBS}

We have a surplus of large-sized Shrubs growing on a piece of ground we wish to clear this spring, and are offering them at the following low prices. They were all cut back and transplanted two years ago and now carry a large ball of roots with numerous branches suitable for making immediate effect, and are all in first-class order.

in first-class order.
Each Spiræa Van Houttei. XX strong clumps, specimens......\$0 25

Deutzia crenata f.-pl. 4 to $6 \mathrm{ft}$. high, specimens......... 25

Deutzia, Pride of Rochester. 4 to $6 \mathrm{ft}$. high, specimens.

3 to $4 \mathrm{ft}$. branches.

piræa Douglasii. 4 feet and over, specimens

3 to $4 \mathrm{ft}$., fine bushy plants

Weigel a arborea grandiflora, Coquette. Desboisii, Ducharte, Gustave Mallet, Groenewegeni, Hendersoni, Symondsii, Stelzneri, Van Houtte, and Eva Rathke. 4 to $6 \mathrm{ft}$. high, specimens..

3 to $4 \mathrm{ft}$. high, stro

Several other varieties of Shrubs in large and small sizes at very reasonable prices. 